\title{
Representación semántica de bullying en niños y niñas mexicanos de edad escolar
}

\section{Semantic representation of bullying in mexican children of school age}

Andrómeda Ivette Valencia Ortiz

Violeta del Carmen Fajardo Vargas

Rubén García Cruz

Norma Angélica Ortega Andrade

Universidad Autónoma del Estado de Hidalgo

\begin{abstract}
Resumen
El creciente aumento de la violencia escolar en niños y niñas mexicanos evidencia la realidad social en que se encuentra el país; los involucrados en el ámbito de los espacios escolares necesitan contar con información en torno a esta problemática con el fin de intervenir en las situaciones con incidencia en el bienestar y la salud de los infantes. En México se han generado algunas alternativas de prevención como de atención en acoso escolar, no obstante, la evaluación de dicho fenómeno descansa en sus principales actores (víctima, agresor), y deja de lado valiosa información percibida por los espectadores de este fenómeno, útil tanto para el avance de la caracterización del fenómeno como para la prevención y la propia intervención. El presente trabajo tuvo como objetivo conocer la percepción de niños y niñas de primaria en torno al acoso escolar, específicamente, el significado que atribuyen a los términos: "bullying" y "aislamiento" como palabras estímulo, así como conocer la percepción de los niños (al usar frases orientadoras) sobre el papel de la víctima, el rol del espectador y la percepción en torno a la supervisión del maestro en el acoso escolar. Se realizó un estudio cualitativo, exploratorio y descriptivo con base en la técnica de redes semánticas naturales. Participaron 208 niños de primaria con un rango de edad entre 8 y 13 años. Los datos obtenidos permitieron clasificar las respuestas sobre las palabras estímulo bullying y aislamiento en tres grandes categorías: violencia física, violencia verbal y violencia psicológica-emocional. Se analizaron y discutieron los resultados y las implicaciones de este estudio.
\end{abstract}

Palabras clave: bullying/acoso escolar, redes semánticas, educación básica, violencia, niños.

Nota del autor

Andrómeda Ivette Valencia Ortiz, Instituto de Ciencias de la Salud (ICS), Universidad Autónoma del Estado de Hidalgo (UAEH); Violeta del Carmen Fajardo Vargas, ICS, UAEH; Rubén García Cruz, ICS, UAEH; Norma Angélica Ortega Andrade, ICS, UAEH.

La correspondencia en relación con este artículo debe dirigirse a Andrómeda Ivette Valencia Ortiz, ICS, UAEH, Ex hacienda La Concepción, carretera Pachuca-Actopan km 4.5, camino a Tilcuautla, colonia Campo de Tiro C. P. 42039, Hidalgo Pachuca, México.

Dirección electrónica: adromeda_valencia@uaeh.edu.mx 


\begin{abstract}
The progressive increase in bullying in Mexican children demonstrates the need for the most accurate information around this problem. Mexico has generated some strategies care prevention in the bullying problem, however, the evaluation of this phenomenon lies in the main actors (victim, attacker), leaving aside valuable information that can provide the viewers of this phenomenon, useful information for the advancement of the characterization of the bullying phenomenon and the prevention and intervention. Hence the importance of the study under this descriptive approach. This study aimed to know the perception of children from elementary school around bullying; specifically the meanings attributed to the terms "bullying" and "isolation" as stimulus words, besides to know the perception of children (guiding phrases) of the role of the victim, the role of the viewer and perception about teacher's supervision in bullying. This was a qualitative, exploratory and descriptive study, using the technique of natural semantic networks. Participants were a group of 208 elementary school children with an age range between 8 and 13 years. The data obtained allowed to classify the stimulus word answers for bullying and isolation in three broad categories: physical violence, verbal violence and psychological-emotional violence. The results and implications of the study were analyzed and discussed.

Keywords: bullying, semantic networks, basic education, violence, children.
\end{abstract}

En los últimos tiempos, la violencia ha sido un fenómeno expuesto al ojo crítico de estudiosos de varias disciplinas, tratan de mediar los conflictos para crear una cultura de paz. La Organización Mundial de la Salud (OMS, 1996) define la violencia como el uso deliberado de la fuerza física o el poder, ya sea en grado de amenaza o efectivo, contra uno mismo, otra persona o un grupo o una comunidad; que cause o tenga muchas probabilidades de causar lesiones, muerte, daños psicológicos, trastornos del desarrollo o privaciones (Concha-Eastman \& Krug, 2002).

Gómez-Palacios desde 1991 plantea educar para la paz como un antídoto contra la violencia, implica una formación que abarca a la totalidad de la persona, como un proceso de inte- riorización y proyección social hacia el entorno territorial y hacia la gran comunidad de la humanidad. Harlem Brundtland directora general de la OMS en el periodo de 1998 al 2003, en el prefacio del informe mundial sobre la violencia y la salud plantea:

La violencia es una constante en la vida de gran número de personas en todo el mundo, y nos afecta a todos de un modo u otro. Para muchos, permanecer a salvo consiste en cerrar puertas y ventanas, y evitar los lugares peligrosos. Para otros no hay escapatoria, porque la amenaza de la violencia está detrás de esas puertas, oculta a los ojos de los demás. $\mathrm{Y}$ para quienes viven en medio de guerras $\mathrm{y}$ conflictos, la violencia impregna todos los aspectos de la vida. (OMS, 2002). 
Según el informe de la OMS (2002), el análisis de la violencia con perspectiva de salud pública ha sido fundamentalmente reactivo $\mathrm{y}$ terapéutico; se tiende a dividir en áreas de interés y de competencias especiales, por lo que es frecuente no realizarlo como un todo integrado y holístico. Sin embargo, es importante recordar la idea propuesta por diversos teóricos desde sus inicios: la violencia como un fenómeno predecible y, por tanto, prevenible. Al respecto, la OMS sugiere como requisito básico para afrontarla en todos los niveles y elaborar respuestas eficaces, trabajar de forma mancomunada entre todas las instituciones para generar cultura de bienestar. Las consecuencias de este fenómeno constituyen indicadores de riesgo relacionados con el sistema de salud, pues se traducen en muertes, homicidios, afectación de los procesos del desarrollo bienestar emocional y calidad de vida; se ha hecho más evidente en los últimos años en todas las esferas sociales de México, incluido el ámbito escolar, espacio de interacción social donde niños y niñas pasan gran parte de sus vidas.

La conducta violenta infantil se ha reconocido como un problema de salud pública a nivel mundial y nacional; la muerte, maltratos y rechazo de la escuela por parte de los infantes al considerarla la consecuencia más tangible de los actos de esta naturaleza. Desde esta perspectiva de salud considerando como un fenómeno predecible y prevenible, Ayala, Pedroza, Morales, Chaparro y Barragán (2002) proponen que los factores de riesgo para el inicio y desarrollo de conducta agresiva en los niños y niñas se pueden clasificar en cuatro grandes grupos: 1) las características inherentes a los niños y las niñas, 2) las características de los padres, 3) los factores contextuales, y 4) la interacción de los padres-hijos. Por otra parte, han clasificado los factores protectores que previenen la conducta antisocial en cinco tipos principales: 1) las relaciones de apoyo padre e hijo, 2) los métodos positivos de disciplina, 3) el monitoreo y la supervisión parental, 4) el involucramiento de los padres en las actividades de los hijos, y 5) la búsqueda de información por parte de los padres, así como posibilidad de solicitar apoyo en la crianza. La familia es, entonces, el espacio en donde se gesta la vulnerabilidad y a la vez los factores de protección (Repetti, Taylor, \& Seeman, 2002).

Desde una visión social se identifica que en América Latina, el desarrollo de sociedades neoliberales proclamadas democráticas, ha marcado grandes diferencias sociales expresadas en cambios culturales, desigualdad, pobreza e incluso carencias de expectativas de futuro. La familia es la primera institución dentro del sistema social que siente el impacto socioeconómico de políticas públicas y, al mismo tiempo, es dicha institución primaria la encargada de brindar protección y apoyo frente a estos cambios. Arriagada (1997), en su estudio sobre los cambios y la desigualdad en las familias latinoamericanas, afirma que las modificaciones de esta institución primaria en el tiempo generan un efecto que provocan en ellas tanto tensiones externas como en las relaciones dentro de su propio seno, lo cual sin duda activa fuentes im- 
portantes de inseguridad interna, cambios en el estado civil (separación, divorcio), migraciones y violencia intrafamiliar. Las familias son vulnerables a las crisis y su impacto afecta otras esferas del contexto social como el propio ambiente escolar.

Pliego (2013) realiza investigaciones comparadas en sociedades democráticas de trece países (Australia, Brasil, Canadá, Chile, Colombia, España, Estados Unidos de América, Holanda, Japón, México, Noruega, Perú y Reino Unido de la Gran Bretaña), analiza la información estadística y censal aportada por 351 estudios, además, relaciona diversas características de la familia con importantes áreas de bienestar. Dicho autor estudia a la familia mexicana a partir de datos comparados en distintos periodos y muestra un cambio social sin precedentes, donde se identifican nuevas dinámicas familiares y el incremento en situaciones de riesgo, principalmente, la presencia de violencia de distintos tipos al interior de las familias (por ejemplo, se eleva a $298 \%$ el abuso sexual en aquellas familias que viven con un padrastro).

Existen diversos estudios entre ellos los antes mencionados, que documentan las variables tanto protectoras como precursoras de la conducta agresiva, no obstante, en los últimos años, el fenómeno de la agresividad infantil sale del contexto familiar y permea otros escenarios sociales. En la actualidad, la conducta violenta se presenta cada vez con mayor frecuencia e intensidad en el contexto escolar. Dichas conductas se desarrollan y mantienen por la inadecuada interacción entre estudiantes, donde destacan algu- nos factores contextuales (Contini de González, 2008). En este mismo sentido, se identifica que el desarrollo de habilidades sociales positivas en la infancia contribuye de forma evolutiva al logro de una personalidad saludable en la adultez; como contrapartida se plantea que la competencia interpersonal pobre se asocia con baja aceptación, rechazo o aislamiento de los pares, con problemáticas escolares y con problemáticas personales, tales como la baja autoestima, la indefensión, inadaptación en la adolescencia, delincuencia juvenil o adicciones (Albores-Gallo, Sauceda-García, Ruiz-Velasco, \& Roque-Santiago, 2011), al igual que la falta de monitoreo y supervisión de los profesores y autoridades escolares aumenta el riesgo de contextos de vulnerabilidad para la presencia de conducta violenta (Pedroza, Mendoza, \& Martínez, 2013).

Otro factor importante consiste en no contar con cifras confiables sobre la frecuencia del problema de la violencia en el ambiente escolar; se debe a razones como (Abdalá, Hernández, Navarrete, Escalera, \& Manuel, 2010):

- No contar con una definición precisa, concreta y clara del fenómeno para ser empleada por todos los profesionales, quienes intervienen en esta patología, de una manera universal.

- No se dispone de un registro nacional.

- La mayoría de los profesionales que interviene en la atención de estos casos tiene un conocimiento muy básico de esta problemática.

- La sociedad tiene una cultura de la denuncia muy precaria. 
Olweus acuñó el término bullying (en inglés) en 1973, para describir el comportamiento agresivo entre pares, caracterizado por: 1) intencionalidad: el agresor emite conductas agresivas con la intención de causar daño o incomodar al otro, 2) persistencia: la conducta agresiva es repetitiva a lo largo del tiempo y no se presenta como un evento aislado, y 3 ) asimetría de poder: entre la víctima y el agresor existen diferencias como fuerza física, edad, habilidades, etcétera. Goodman, Ford y Richards (2000) lo caracterizan como el uso repetido y deliberado de agresiones verbales, psicológicas o físicas para lastimar y dominar a otro niño o niña, sin que hayan sido precedidas de provocación y en el conocimiento de que la víctima carece de posibilidades de defenderse. Los participantes en el acoso escolar se pueden clasificar en cuatro categorías: agresor (bully), víctima, víctimaagresor, y el neutro (compañero no implicado en el fenómeno de acoso). La mayor parte del acoso ocurre en la escuela, más que en el camino a ella o de regreso de ella. Los agresores (bullies) y las víctimas generalmente están en el mismo grado escolar y, aunque se supone que los menores son supervisados en la escuela, gran proporción de los episodios de acoso no son del conocimiento de los maestros y la víctima habitualmente se siente incapaz de reportarlos a éstos y a sus padres.

En ese mismo sentido, el acoso, maltrato, hostigamiento e intimidación entre escolares, se ha identificado como una conducta de persecución física o psicológica que realiza el alumno o la alumna contra otro, a quien elige como víctima de repetidos ataques. Esta acción, negativa e intencionada, sitúa a las víctimas en posiciones de las cuales difícilmente pueden salir por sus propios medios. A estas características descritas por Olweus, se han agregado otros componentes como la vulnerabilidad de la víctima, la falta de apoyo, el aislamiento de la víctima y la presencia de consecuencias a corto y largo plazo (Manesini, 1997; Farrington, 1993; Fried \& Fried, 1996; Hunter \& Boyle, 2004; Olweus, 1993; Olweus, 2001; Porcelli \& Urpis, 2012).

En situaciones de bullying se observa la interacción del agresor y la víctima, además de la participación del espectador(es), quienes adoptan distintos roles como: a) Permanecer neutral durante la situación, b) Ayudar y apoyar al agresor, c) Apoyar o consolar a la víctima. Desafortunadamente, en la mayoría de los casos, el rol que adopta el observador(es) es el de apoyar al agresor para continuar con el acoso (Aluede, Adeleke, Omoike, \& Afen-Akpaida, 2008; Coloroso, 2004; Elizalde, 2010; Farrington, 1993; Olweus, 1993; Olweus, 2001). En la figura 1 se puede observar gráficamente la dinámica del bullying entre escolares. 


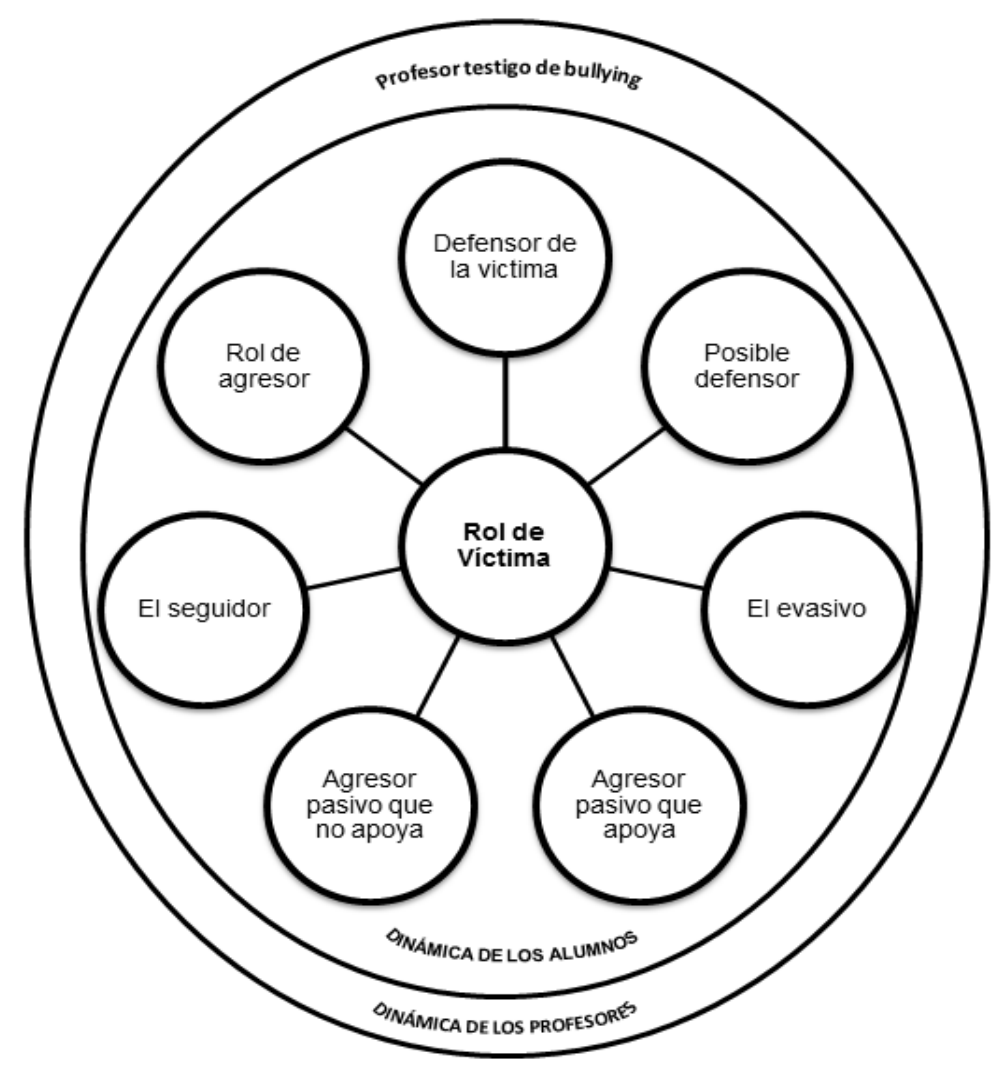

Figura 1. Dinámica del bullying. Adaptada de Elizalde (2010).

Sin embargo, los estudios de esta problemática se han centrado en su mayoría en las características de las víctimas, dejando en segundo plano a los agresores, y la mayoría de las veces olvidando los otros roles involucrados en la dinámica del bullying. Westhues (junio, 2008) afirma que gran parte de los investigadores del bullying, sistemáticamente, privilegian las quejas de las presuntas víctimas y concluye que el estudio científico de este fenómeno, como en cualquier tipo de conflicto, requiere que el investigador dude de las demandas de ambas partes y examine de manera independiente y objetiva los hechos de lo que está pasando.
De acuerdo a Barba-Solano (2010), los datos de la Encuesta Nacional sobre Discriminación en México (ENADIS- 2010) informan que 12.7 $\%$ de las niñas y los niños respondieron que sus compañeros les habían pegado alguna vez en los meses previos a la entrevista. En la encuesta se muestra que esta situación se presenta en mayor proporción entre los niños (13.7\%) que entre las niñas (11.5\%). Dichos datos parecen apuntar que los niños están más expuestos al maltrato físico por parte de sus compañeros de escuela en comparación con las niñas. Sin embargo, existen otros actos de intimidación o victimización en los cuales se reportan mayores porcentajes entre 
las niñas, por ejemplo: avergonzar a otros niños, (9.7 \% para niñas y $6.1 \%$ para niños); no invitar a hacer algo juntos (28\% para niñas y $24.9 \%$ para niños).

Por otro lado, al intentar caracterizar el fenómeno de bullying en un ámbito más amplio, se realizó un estudio para conocer la relación entre algunos trastornos psiquiátricos y la frecuencia del acoso escolar. Se encontró que existen mayores índices de psicopatología en las víctimas-agresores (aquellos niños que han estado en las dos posiciones, tanto de víctimas como de agresores) y los que son víctimas. Estos últimos mostraron mayores puntajes en la escala de ansiedad. La muestra estuvo conformada por 1,092 niños de la Ciudad de México, quienes cursaban desde segundo hasta sexto grado de primaria. De la muestra total, $8.9 \%$ fue categorizado como víctima, $12.2 \%$ como agresor, 2.7 \% como víctima-agresor y $76 \%$ como control (Albores-Gallo et al., 2011).

Según datos de la segunda Encuesta Nacional de Exclusión, Intolerancia y Violencia en las Escuelas Públicas de Educación Media Superior (ENEIVEMS, 2009) el acoso escolar trasciende todos los niveles de educación básica. Como resultado de la encuesta se encontró que $76.4 \%$ de los adolescentes que cursaban la educación media superior, (con un rango de edad entre los 15 y 17 años), declaró haber tenido por lo menos un episodio de maltrato físico, exclusión, humillación o intimidación por parte de sus compañeros. Dentro de este tipo de conducta de acoso, $81 \%$ de los hombres informó haberlo su- frido, mientras que en las mujeres el porcentaje fue $72.9 \%$, aunque ambas cifras son elevadas la diferencia muestra que existe mayor presencia de acoso en los varones, sin embargo, es importante conocer las estrategias de afrontamiento para identificar mejor a qué se atribuyen estos datos, pues es probable que el rol de género marque de forma importante las pautas de conducta ante el acoso escolar.

Dado el creciente número de niños y adolescentes que viven situaciones de acoso escolar en México (reflejado en las encuestas y estudios dedicados a documentar dicho fenómeno), se han generado en los últimos años, estrategias oficiales para generar pautas de convivencia entre escolares. Ejemplo de ello es el Oficio-Circular de la Administración Federal de Servicios Educativos en el Distrito Federal No. AFSEDF/642/2011, de fecha 28 de octubre de 2011, mediante el cual se publicaron los Lineamientos Generales por los que se establece un Marco para la Convivencia Escolar en las Escuelas de Educación Básica del Distrito Federal (Administración Federal de Servicios Educativos en el Distrito Federal, 2011); representa un esfuerzo importante por atender el problema, aunque insuficiente, pues aún no permea a toda la federación, y acentúa más las diferencias ya existentes entre las políticas públicas de educación entre los estados de la República Mexicana.

Una cuestión particularmente interesante con respecto a la caracterización del fenómeno de acoso escolar tiene relación con las estrategias utilizadas para su evaluación. Entre los méto- 
dos de evaluación comúnmente utilizados se encuentran narraciones y representaciones pictóricas (realizadas por los propios alumnos), entrevistas, viñetas, preguntas aisladas, dibujos, fotografías, videos, cuestionarios, cuestionarios sociométricos y autoinformes aplicados en su mayoría a alumnos (víctimas y/o agresores). Los estudios han dedicado menor atención a los otros involucrados en el fenómeno de bullying como es el caso de los espectadores y los maestros (Marín-Martínez \& Reidl-Martínez, 2013). Por lo tanto, es indispensable incluir a todos los actores involucrados con el objetivo de entender la problemática y generar responsabilidad social, para no dejar este fenómeno como una preocupación solo de la comunidad escolar.

Recientemente, los espectadores han cobrado un papel importante con respecto a la formulación de propuestas de intervención en acoso escolar. Algunos autores proponen que resulta más efectivo enfocar la intervención al integrar a todos los niños del grupo, en especial a los espectadores, para que sean éstos los que dialoguen y determinen entre sí las consecuencias para los acosadores en vez de simplemente dejar dichas decisiones en manos de los adultos (maestros o directivos), tal como se propone en la metodología para la reflexión grupal de los sucesos escolares, conocida como "asambleas escolares". Estas estrategias pretenden resolver conflictos, al permitir que los niños experimenten la democracia, la honestidad, la comunicación asertiva, pero, sobre todo, la honestidad para aceptar y hacerse cargo de sus actos (Avedaño \& Minujin, 1988; Mendoza, 2014).
Dada la importancia de involucrar a los otros actores (espectadores) en la intervención del acoso escolar y dada la escasez de información referente a la percepción de los mismos y a la percepción sobre el papel que deben asumir en el cada vez más extendido fenómeno de acoso escolar, se planteó el presente estudio con la intención de aportar información sobre la percepción de niños mexicanos en torno al problema de acoso escolar entre compañeros. El propósito general del presente estudio fue conocer la percepción de niños y niñas de primaria en torno al acoso escolar, específicamente el significado que atribuyen a los términos: bullying y aislamiento como palabras estímulo, así como conocer la percepción de los niños (usando frases orientadoras) sobre el papel de la víctima, el rol del espectador y la percepción en torno a la supervisión del maestro en el acoso escolar.

\section{Método}

\section{Participantes}

En la primera parte del estudio participó un total de 208 niños $(n=110)$ y niñas $(n=98)$, que se encontraba cursando la primaria pública al sur de la Ciudad de México, en los grados de tercero (25.9\%), cuarto $(29.3 \%)$, quinto (21.6 $\%$ ) y sexto de primaria $(23.1 \%)$, respectivamente. Con un rango de edad de los 8 a los 13 años de edad y una media de 10 años de edad.

\section{Instrumento}

Se realizó un estudio cualitativo, interpretetativo donde se dieron categorías (estímulos) relacionadas con la palabra bullying, estas palabras 
se sometieron al análisis de redes semánticas naturales. El valor de esta estrategia reside en que las taxonomías obtenidas se generan de manera directa de la memoria semántica del sujeto y el orden otorgado va de acuerdo con su escala de valores y percepciones. Cuando se le pide al sujeto que mencione las palabras que definen el concepto (palabra estímulo), éste utilizará su memoria y seleccionará aquellas que asume están más relacionadas. En este sentido, la elección es resultado de un proceso subjetivo de representarse al mundo, en particular a la palabra estímulo (Zermeño, Arellano, \& Ramírez, 2005).

\section{Procedimiento}

Para el uso de redes semánticas naturales se seleccionaron palabras estímulo con la pretensión de conocer el significado que le dan los sujetos miembros de algún grupo en particular. A estos se les pidió que definieran la palabra estímulo mediante un mínimo de cinco palabras sueltas, desde verbos, adverbios, adjetivos, sustantivos hasta pronombres, sin utilizar artículos ni preposiciones. Cuando los participantes ya tenían su lista de palabras definidoras, se les pidió que, de manera individual, las jerarquizaran de acuerdo con la cercanía o importancia que cada una de las palabras tenía con la palabra estímulo. Después, asignaron el número uno a la palabra más cercana al estímulo, el dos a la siguiente y así sucesivamente, hasta agotar todas las palabras definidoras. Luego se realizó una relación de las palabras dichas y se elaboró una tabla con todas las palabras definidoras que fueron usadas, para calcular los principales va- lores de la red semántica (Principalmente, el valor $\mathrm{M}$, que corresponde al peso semántico de cada palabra definidora; Hinojosa, 2008).

Se trabajó por grado escolar: se entregó a cada niño participante una hoja con las palabras estímulo bullying y aislamiento, se les indicó que escribieran la primera palabra que en su opinión describiera mejor cada estímulo y después las jerarquizaran colocando el número 1 a la que consideraban la palabra más relacionada con la palabra estímulo. Posteriormente, en la misma hoja se presentaron tres frases orientadoras: 1) Cuando veo que molestan a otro niño debo quedarme callado, 2) Mi maestra hace algo cuando molestan a un niño, 3) Otros niños me molestan. Los niños eligieron una de cuatro opciones de respuesta (escala tipo Likert) que iban desde nunca hasta siempre.

\section{Resultados}

Para la descripción y análisis de variables cualitativas, se utilizaron tablas de contingencia, considerando que los resultados obtenidos con las palabras estímulo fueron clasificados según su contenido en tres categorías (como se observa en la figura 2). La categoría que obtuvo mayor frecuencia fue la de violencia física (79.79\%), seguida de violencia verbal $(8.64 \%)$ y violencia psicológica-emocional (8.64\%), respectivamente. 


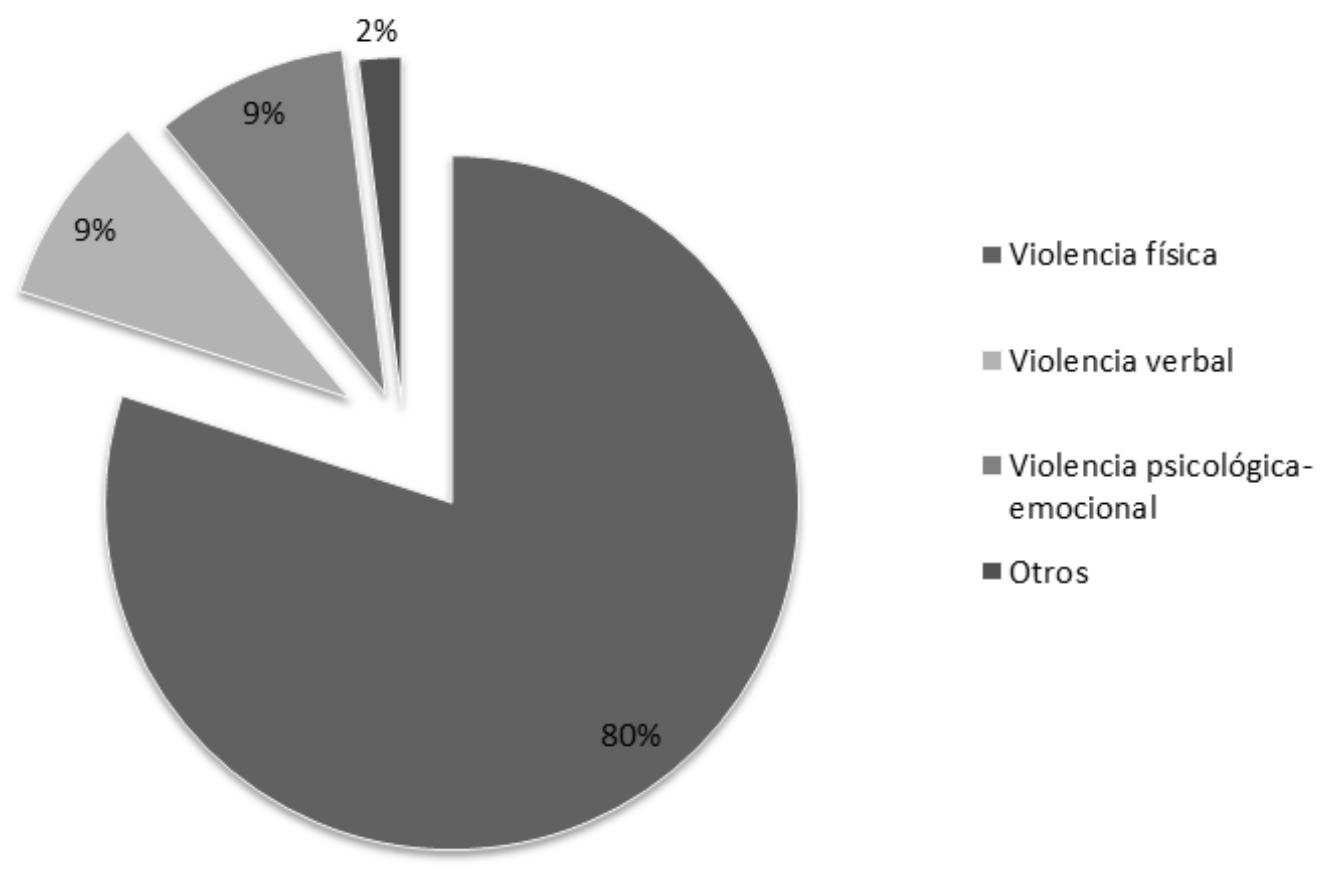

Figura 2. Principales categorías de los descriptores del estímulo bullying.

\section{Análisis de palabras estímulo}

La tabla 1 muestra relación entre la variable de grado escolar y la categoría de violencia física para la palabra estímulo bullying; se encontró que el principal descriptor fue la palabra agresión, con una frecuencia equivalente a $31.25 \%$ de la muestra, según información, principalmente, de alumnos de cuarto de primaria, seguidos de alumnos de quinto, primero y sexto, respectivamente. El segundo descriptor más utilizado (13.96 \%) fue la palabra golpes, mencionado por igual por los alumnos de cuarto y sexto, seguidos de alumnos de quinto y primero. El tercer descriptor fue la palabra violencia con un porcentaje de $11.53 \%$, informado principalmente por alumnos de sexto, seguidos por igual por estudiantes de cuarto y quinto sin ser mencionado por alumnos de tercero. A pesar de la baja frecuencia es importante mencionar que en sexto de primaria se informó el descriptor de abuso sexual y pegar hasta matar, algo similar a lo observado en tercero de primaria donde se encontró el descriptor de matar y violar (como se observa en la tabla 1).

Con respecto a la relación entre la variable de grado escolar y la categoría de violencia verbal para la palabra estímulo bullying, el principal descriptor fue la palabra burlas con un porcentaje de $2.88 \%$, informado igual por alumnos de tercero y quinto, seguido de alumnos de cuarto y sexto. El segundo descriptor fue la palabra violencia verbal, sólo utilizado por alumnos de sexto, como se puede observar en la tabla 2. 
Tabla 1

Contingencia por grado escolar y categoría de violencia física

\begin{tabular}{|c|c|c|c|c|c|c|c|c|}
\hline Categoría & Descriptor & Tercero & Cuarto & Quinto & Sexto & Frecuencia & Porcentaje & $\begin{array}{c}\text { Valor general } \\
\qquad \mathrm{M}\end{array}$ \\
\hline \multirow{23}{*}{$\begin{array}{l}\text { Violencia } \\
\text { física }\end{array}$} & Abuso & 0 & 0 & 1 & 0 & 1 & .48 & 10 \\
\hline & Abuso Sexual & 0 & 0 & 0 & 1 & 1 & .48 & 10 \\
\hline & Agresión & 10 & 26 & 20 & 6 & 65 & 31.25 & 650 \\
\hline & Asaltar & 1 & 0 & 0 & 0 & 1 & .48 & 10 \\
\hline & Contacto físico & 1 & 0 & 0 & 3 & 4 & 1.92 & 40 \\
\hline & Desangrar & 0 & 1 & 0 & 0 & 1 & .48 & 10 \\
\hline & Empujar & 2 & 0 & 0 & 0 & 2 & .96 & 20 \\
\hline & Golpes & 2 & 11 & 5 & 11 & 29 & 13.96 & 290 \\
\hline & Lastimarnos & 0 & 2 & 0 & 0 & 2 & .96 & 20 \\
\hline & Luchadores & 1 & 0 & 0 & 0 & 1 & .48 & 10 \\
\hline & Maltrato & 0 & 0 & 1 & 1 & 2 & .96 & 20 \\
\hline & Matar & 4 & 0 & 0 & 0 & 4 & 1.92 & 40 \\
\hline & $\begin{array}{l}\text { Me agreden mis } \\
\text { compañeros }\end{array}$ & 0 & 0 & 0 & 1 & 1 & .48 & 10 \\
\hline & Molestar & 1 & 0 & 0 & 2 & 3 & 1.44 & 30 \\
\hline & Montón (echar) & 0 & 0 & 1 & 0 & 1 & .48 & 10 \\
\hline & Pegar & 9 & 3 & 0 & 1 & 13 & 6.25 & 130 \\
\hline & $\begin{array}{l}\text { Pegar hasta } \\
\text { matar }\end{array}$ & 0 & 0 & 0 & 1 & 1 & .48 & 10 \\
\hline & Pelear & 5 & 2 & 0 & 0 & 7 & 3.36 & 70 \\
\hline & Robar & 1 & 0 & 0 & 0 & 1 & .48 & 10 \\
\hline & Violar & 2 & 0 & 0 & 0 & 2 & .96 & 20 \\
\hline & Violencia & 0 & 7 & 7 & 10 & 24 & 11.53 & 240 \\
\hline & Total & 39 & 52 & 35 & 37 & 166 & - & \\
\hline & Porcentaje & 19 & 25 & 17 & 18 & - & 79.79 & \\
\hline
\end{tabular}

Tabla 2

Tabla de contingencia por grado escolar y categoría de violencia verbal

\begin{tabular}{llccccccc}
\hline Categoría & Descriptor & Tercero & Cuarto & Quinto & Sexto & Frecuencia & Porcentaje & $\begin{array}{c}\text { Valor general } \\
\text { M }\end{array}$ \\
\hline \multirow{5}{*}{ Amenazar } & 2 & 0 & 0 & 0 & 2 & .96 & 20 \\
& Burlas & 2 & 1 & 2 & 1 & 6 & 2.88 & 60 \\
Violencia & Gritar & 1 & 0 & 0 & 0 & 1 & .48 & 10 \\
verbal & Groserías & 2 & 0 & 0 & 0 & 2 & .96 & 20 \\
& Insulto & 0 & 0 & 1 & 1 & 2 & .96 & 20 \\
& Ofensa & 0 & 0 & 1 & 0 & 1 & .48 & 10 \\
& Violencia & 0 & 0 & 0 & 4 & 4 & 1.92 & 40 \\
& verbal & & & & & & & - \\
\hline & Total & 7 & 1 & 4 & 6 & 18 & - & \\
& Porcentaje & 3.36 & .48 & 1.92 & 2.88 & - & 8.64 & \\
\hline
\end{tabular}


En la tabla 3, se observa la relación entre la variable de grado escolar y la categoría de violencia psicológica-emocional para la palabra estímulo bullying. Se identificó que el principal descriptor fue miedo (1.44\%), informada en todos los grados menos tercero. Seguido de los descriptores discriminar (reportado por cuarto y sexto) y malos sentimientos (mencionado en cuarto y quinto), ambos descriptores con el mismo porcentaje $(.96 \%)$.

Tabla 3

Contingencia por grado escolar y categoría de violencia psicológica-emocional

\begin{tabular}{|c|c|c|c|c|c|c|c|c|}
\hline Categoría & Descriptor & Tercero & Cuarto & Quinto & Sexto & Frecuencia & Porcentaje & $\begin{array}{c}\text { Valor general } \\
M\end{array}$ \\
\hline \multirow{16}{*}{$\begin{array}{l}\text { Violencia } \\
\text { psicológica- } \\
\text { emocional }\end{array}$} & $\begin{array}{l}\text { Algunos tienen } \\
\text { odio entre sí }\end{array}$ & 0 & 0 & 1 & 0 & 1 & .48 & 10 \\
\hline & Discriminar & 0 & 1 & 0 & 1 & 2 & .96 & 20 \\
\hline & Gozar & 0 & 1 & 0 & 0 & 1 & .48 & 10 \\
\hline & $\begin{array}{l}\text { Estar discapaci- } \\
\text { tado }\end{array}$ & 0 & 0 & 0 & 1 & 1 & .48 & 10 \\
\hline & Humillación & 0 & 0 & 0 & 1 & 1 & .48 & 10 \\
\hline & $\begin{array}{l}\text { Malos sentimien- } \\
\text { tos }\end{array}$ & 0 & 1 & 1 & 0 & 2 & .96 & 20 \\
\hline & Miedo & 0 & 1 & 1 & 1 & 3 & 1.44 & 30 \\
\hline & Peligroso & 1 & 0 & 0 & 0 & 1 & .48 & 10 \\
\hline & $\begin{array}{l}\text { Problemas de } \\
\text { personas enoja- } \\
\text { das }\end{array}$ & 0 & 0 & 1 & 0 & 1 & .48 & 10 \\
\hline & Por respeto & 1 & 0 & 0 & 0 & 1 & .48 & 10 \\
\hline & $\begin{array}{l}\text { Sentirse "muy } \\
\text { muy" }\end{array}$ & 0 & 0 & 1 & 0 & 1 & .48 & 10 \\
\hline & Sentirse jefe & 0 & 1 & 0 & 0 & 1 & .48 & 10 \\
\hline & Tristeza & 0 & 0 & 0 & 1 & 1 & .48 & 10 \\
\hline & Videos & 1 & 0 & 0 & 0 & 1 & .48 & 10 \\
\hline & Total & 3 & 5 & 5 & 5 & 18 & - & 180 \\
\hline & Porcentaje & 1.44 & 2.4 & 2.4 & 2.4 & - & 8.64 & \\
\hline
\end{tabular}

Sólo $17.29 \%$ de la población respondió a la palabra estímulo de aislamiento, al identificar que la categoría con mayor frecuencia de respuestas fue violencia psicológica-emocional $(8.64 \%)$, seguida de la categoría de violencia física (7.21
$\%)$, mientras que para la categoría de violencia verbal el porcentaje fue mínimo (1.44\%).

Los resultados de la relación entre la variable de grado escolar y la categoría de violencia psicológica-emocional muestran que el descriptor más uti- 
lizado es la palabra soledad con un porcentaje de $3.84 \%$, informado principalmente por alumnos de tercero. El segundo descriptor corresponde a la palabra triste $(2.40 \%)$ de acuerdo con los alumnos de tercero y cuarto (tabla 4).
Para la categoría de violencia física y su relación con la variable de grado escolar, se identificó como principal descriptor: fue cuando una persona aísla a otra (1.44\%), informado únicamente por niños de tercero (tabla 5).

\section{Tabla 4}

Tabla de contingencia por grado escolar y categoría de violencia psicológica-emocional para el estímulo aislamiento

\begin{tabular}{|c|c|c|c|c|c|c|c|c|}
\hline Categoría & Descriptor & Tercero & Cuarto & Quinto & Sexto & Frecuencia & Porcentaje & $\begin{array}{c}\text { Valor general } \\
M\end{array}$ \\
\hline \multirow{8}{*}{$\begin{array}{l}\text { Violencia } \\
\text { psicológica- } \\
\text { emocional }\end{array}$} & Asustar & 0 & 1 & 0 & 0 & 1 & .48 & 10 \\
\hline & Miedo & 2 & 0 & 0 & 0 & 2 & .96 & 20 \\
\hline & $\begin{array}{l}\text { Nadie me } \\
\text { quiere }\end{array}$ & 1 & 0 & 0 & 0 & 1 & .48 & 10 \\
\hline & $\begin{array}{l}\text { No aguan- } \\
\text { tarse }\end{array}$ & 1 & 0 & 0 & 0 & 1 & .48 & 10 \\
\hline & Soledad & 5 & 3 & 0 & 0 & 8 & 3.84 & 80 \\
\hline & Triste & 3 & 2 & 0 & 0 & 5 & 2.40 & 50 \\
\hline & Total & 12 & 6 & 0 & 0 & 18 & - & 180 \\
\hline & Porcentaje & 5.76 & 2.88 & 0 & 0 & - & 8.64 & \\
\hline
\end{tabular}

Tabla 5

Tabla de contingencia por grado escolar y categoría de violencia física para el estímulo aislamiento

\begin{tabular}{llccccccc}
\hline Categoría & Descriptor & Tercero & Cuarto & Quinto & Sexto & Frecuencia & $\begin{array}{c}\text { Porcentaje } \\
\text { Valor general } \\
\text { M }\end{array}$ \\
\hline \multirow{5}{*}{$\begin{array}{l}\text { Cuando no juegan } \\
\text { conmigo }\end{array}$} & 2 & 0 & 0 & 0 & 2 & .96 & 20 \\
& $\begin{array}{l}\text { Cuando una per- } \\
\text { sona aísla a otra }\end{array}$ & 3 & 0 & 0 & 0 & 3 & 1.44 & 30 \\
& Empujar & 2 & 0 & 0 & 0 & 2 & .96 & 20 \\
Violencia & Golpear & 0 & 1 & 0 & 0 & 1 & .48 & 10 \\
física & Dejar solo & 1 & 1 & 0 & 0 & 2 & .96 & 20 \\
& Matar & 1 & 0 & 0 & 0 & 1 & .48 & 10 \\
& Molestar & 0 & 1 & 0 & 0 & 1 & .48 & 10 \\
& No hacer caso & 1 & 0 & 0 & 0 & 1 & .48 & 10 \\
& No salir & 0 & 1 & 0 & 0 & 1 & .48 & 10 \\
& Tirar & 1 & 0 & 0 & 0 & 1 & .48 & 10 \\
\hline & Total & 11 & 4 & 0 & 0 & 15 & - & 150 \\
& Porcentaje & 5.28 & 1.92 & 0 & 0 & - & 7.21 & \\
\hline
\end{tabular}


La tabla 6, de contingencia por grado escolar y la categoría de violencia verbal para el estímulo aislamiento, muestra el descriptor decir groserías como el principal, sin embargo, su porcentaje de aparición es muy bajo (.96\%).

\section{Análisis de frases orientadoras}

Al realizar el análisis de la frase orientadora "Cuando veo que molestan a otro niño debo quedarme callado", se observa que $80.30 \%$ de los niños seleccionó la opción nunca, sin embargo, la siguiente opción de respuesta en frecuencia fue siempre con $8.08 \%$, como se puede observar en la tabla 7.

Para la frase orientadora "Mi maestra hace algo cuando molestan a un niño", el $61.42 \%$, mencionó que siempre, o muchas veces (16.75 $\%$ ), mientras que el $12.18 \%$ informó que pocas veces y $9.64 \%$ dijo que nunca.

Tabla 6

Tabla de contingencia por grado escolar y categoría de violencia verbal para el estímulo aislamiento

\begin{tabular}{llccccccc}
\hline Categoría & Descriptor & Tercero & Cuarto & Quinto & Sexto & Frecuencia & Porcentaje & $\begin{array}{c}\text { Valor general } \\
\text { M }\end{array}$ \\
\hline Violencia & Amenazar & 1 & 0 & 0 & 0 & 1 & .48 & 10 \\
verbal & Decir groserías & 1 & 1 & 0 & 0 & 2 & .96 & 20 \\
\hline \multirow{2}{*}{} & Total & 2 & 1 & 0 & 0 & 3 & - & 30 \\
& Porcentaje & .96 & .48 & 0 & 0 & - & 1.44 & \\
\hline
\end{tabular}

Tabla 7

Tabla de contingencia por grado escolar para la afirmación "Cuando veo que molestan a otro niño debo quedarme callado"

\begin{tabular}{|c|c|c|c|c|c|c|c|}
\hline & & \multicolumn{4}{|c|}{ Grado } & \multirow{2}{*}{ Frecuencia } & \multirow{2}{*}{ Porcentaje } \\
\hline & & Tercero & Cuarto & Quinto & Sexto & & \\
\hline \multirow{4}{*}{$\begin{array}{l}\text { Cuando veo que } \\
\text { molestan a otro niño } \\
\text { debo quedarme } \\
\text { callado }\end{array}$} & Nunca & 28 & 45 & 39 & 45 & 159 & 80.30 \\
\hline & $\begin{array}{l}\text { Pocas } \\
\text { veces }\end{array}$ & 5 & 6 & 2 & 1 & 14 & 7.07 \\
\hline & $\begin{array}{l}\text { Muchas } \\
\text { veces }\end{array}$ & 4 & 3 & 1 & 1 & 9 & 4.54 \\
\hline & Siempre & 5 & 7 & 2 & 1 & 16 & 8.08 \\
\hline \multicolumn{2}{|l|}{ Total } & 42 & 61 & 44 & 48 & 198 & 100 \\
\hline
\end{tabular}

Nota: 10 participantes no contestaron por lo que se consideró la $\mathrm{n}=198$ para este análisis. 
Finalmente, para la frase orientadora de “Otros niños me molestan" se encontró que $17.25 \%$ de los niños encuestados informó que siempre los molestan, o muchas veces con un porcentaje de $14.21 \%$, mientras que $49.74 \%$ informó que pocas veces lo molestan. Solo 18.78 $\%$ mencionó que nunca lo molestan (ver tabla 9).

Tabla 8

Tabla de contingencia por grado escolar para la afirmación "Mi maestra hace algo cuando molestan a un niño"

\begin{tabular}{|c|c|c|c|c|c|c|c|}
\hline & & \multicolumn{4}{|c|}{ Grado } & \multirow{2}{*}{ Frecuencia } & \multirow{2}{*}{ Porcentaje } \\
\hline & & Tercero & Cuarto & Quinto & Sexto & & \\
\hline \multirow{4}{*}{$\begin{array}{l}\text { Mi maestra hace } \\
\text { algo cuando mo- } \\
\text { lestan a un niño }\end{array}$} & Nunca & 7 & 5 & 4 & 3 & 19 & 9.64 \\
\hline & $\begin{array}{l}\text { Pocas } \\
\text { veces }\end{array}$ & 8 & 10 & 5 & 1 & 24 & 12.18 \\
\hline & $\begin{array}{l}\text { Muchas } \\
\text { veces }\end{array}$ & 7 & 13 & 12 & 1 & 33 & 16.75 \\
\hline & Siempre & 21 & 33 & 23 & 43 & 121 & 61.42 \\
\hline Total & & 43 & 61 & 44 & 48 & 197 & 100 \\
\hline
\end{tabular}

Nota: 11 participantes no contestaron por lo que se consideró la $n=197$ para este análisis.

Tabla 9

Tabla de contingencia por grado escolar para la afirmación "Otros niños me molestan"

\begin{tabular}{|c|c|c|c|c|c|c|c|}
\hline & & \multicolumn{4}{|c|}{ Grado } & \multirow{2}{*}{ Frecuencia } & \multirow{2}{*}{ Porcentaje } \\
\hline & & Tercero & Cuarto & Quinto & Sexto & & \\
\hline \multirow{4}{*}{$\begin{array}{l}\text { Otros niños me } \\
\text { molestan }\end{array}$} & Nunca & 7 & 9 & 11 & 10 & 37 & 18.78 \\
\hline & $\begin{array}{l}\text { Pocas } \\
\text { veces }\end{array}$ & 15 & 29 & 24 & 30 & 98 & 49.74 \\
\hline & $\begin{array}{l}\text { Muchas } \\
\text { veces }\end{array}$ & 10 & 8 & 4 & 6 & 28 & 14.21 \\
\hline & Siempre & 12 & 15 & 5 & 2 & 34 & 17.25 \\
\hline \multicolumn{2}{|c|}{ Total } & 45 & 61 & 44 & 48 & 197 & 100 \\
\hline
\end{tabular}

Nota: 11 participantes no contestaron por lo que se consideró la $n=197$ para este análisis. 


\section{Discusión}

La situación de cambio en las familias y el incremento en episodios de violencia familiar, así como los problemas sociales que nos aquejan cotidianamente han sido, sin lugar a dudas, el caldo de cultivo propicio para que la violencia llegue a las aulas y forme parte de la vida cotidiana de niños y niñas que ahora tienen dentro de su contexto de crecimiento, situaciones de acoso escolar a las cuales tienen que hacer frente. El problema del bullying o acoso entre pares tiene graves repercusiones para la víctima, el agresor y los observadores. Sin embargo, parte importante para el desarrollo de estrategias de intervención es conocer la percepción que tienen los involucrados en la problemática a tratar.

A pesar de las claras limitaciones de los estudios descriptivos, los datos encontrados en este estudio permiten observar que los estudiantes asocian principalmente el bullying con agresión física. Por lo tanto, es probable que se requiera de mayor sensibilización ante otras formas de agresión entre pares, como la agresión verbal o la emocional-psicológica. Es importante enfocar nuestros esfuerzos a disminuir el riesgo de que niños y niñas consideren aceptables estas conductas de agresión, solo por el hecho de no llegar a una conducta física.

En este mismo sentido, llama la atención que alumnos de tercero y sexto de primaria reporten descriptores como golpear hasta matar, matar o violar para la descripción del bullying, pues ya no solo se identifican como golpes o agresiones menores, sino como dos de las situaciones más relacionadas con conductas antisociales, como el abuso sexual y el privar de la vida a un ser humano. En las respuestas entre estos dos grupos se puede observar la relación con la etapa de desarrollo en la que se encuentran; es relevante rescatar esta información, ya que puede servir como predictor - a comprobar- del tipo e intensidad del bullying en la secundaria. Esto favorecería el desarrollo de estrategias de prevención o intervención más adecuadas a la percepción y etapa del desarrollo de los niños.

Al pedir a los alumnos que describieran la palabra estímulo aislamiento, solo un porcentaje bajo respondió, por lo cual es importante conocer cuál es la razón de que en quinto y sexto de primaria esta palabra no tuvo mayor relevancia.

Con respecto a las frases orientadoras al presentar el estímulo "Cuando veo que molestan a otro niño debo quedarme callado", $80.30 \%$ de los participantes en el estudio seleccionó la opción de nunca, lo cual permite identificar que consideran deseable que no guarden silencio ante una situación de bullying, sin embargo, la siguiente opción con mayor frecuencia indica el extremo contrario siempre $(8.08 \%)$.

A pesar del porcentaje que respondió siempre, menor $10 \%$, todo indica que hay un grupo de estudiantes vulnerables, que permitiría se presentara una situación de agresión frente a ellos, ya sea porque se encuentra en el papel de agresores, víctima, o bien, como observador.

Finalmente, el informe de los participantes es muy similar a lo encontrado por la segunda Encuesta Nacional de Exclusión, Intolerancia y Violencia en las Escuelas Públicas de Educación Media Superior (ENEIVEMS, 2009), quienes encontraron que $76.4 \%$ de los adolescentes que 
cursaban la educación media superior (entre 15 y 17 años), había tenido por lo menos un episodio de maltrato físico, exclusión, humillación o intimidación por parte de sus compañeros. Los datos de este estudio informan que al mostrarles la frase orientadora "Otros niños me molestan", $81.2 \%$ ha sido molestado (desde siempre, muchas veces o pocas veces), y solo $17.25 \%$ de los niños encuestados quienes informa que nunca lo ha molestado. Resulta muy relevante saber que lo informado por adolescentes muy probablemente inició en la primaria.

Las intervenciones preventivas-selectivas deben ser una opción de trabajo en las escuelas primarias, de tal manera que los estudiantes, niños y niñas, tengan la oportunidad de conocer las diferentes formas de agresión, estén sensibilizados a las alternativas no violentas de respuesta y, sobre todo, que el contexto de los adultos en el ámbito escolar favorezca el crecimiento y desarrollo integral de los niños, incluyendo sus habilidades sociales y la capacidad de resolver problemas sin agresiones.

De igual manera, el uso de las asambleas escolares permitiría encontrar un espacio propicio para la reflexión de los propios niños y de esta manera cambiaría su percepción sobre las conductas de riesgo, por lo cual también se pueden incrementar sus acciones de prevención. Por consiguiente, es necesaria la capacitación y psico-profesionalización de los maestros y directivos como facilitadores del cambio de percepción y manejo de niños y niñas en situaciones de conflicto, como una excelente estrategia de prevención-acción.

\section{Referencias}

Abdalá, A. L., Hernández, J. T., Piña, C. G., González, A. P., Navarrete, G. E. L., Escalera, P. M. I. A., \& Manuel, J. (2010). Consenso de la Comisión para el Estudio y Atención Integral al Niño Maltratado. Maltrato infantil: Una acción interdisciplinaria e interinstitucional en México. Acta Pediátrica de México, 31(1).

Administración Federal de Servicios Educativos en el Distrito Federal (2011). Lineamientos Generales por los que se establece un Marco para la Convivencia Escolar en las Escuelas de Educación Básica del Distrito Federal. Recuperado de http:/www.sepdf.gob.mx/ principal/archivos/Lineamientos_Grales_EscuelasEducBasica.pdf

Albores-Gallo, L., Sauceda-García, J. M., RuizVelasco, S., \& Roque-Santiago, E. (2011). El acoso escolar (bullying) y su asociación con trastornos psiquiátricos en una muestra de escolares en México. Salud Pública de México, 53(3), 220-227.

Aluede, O., Adeleke, G., Omoike, D., \& AfenAkpaida, J. (2008). A Review of the Extent, Nature, Characteristics and Effects of Bullying Behaviour in Schools. Journal of Instructional Psychology, 35(2), 151-158.

Anderson, M., Kaufman, J., \& Simon, T. R. (2001). School-associated violent deaths in the United States. 1994-1999. JAMA, 286, 2695-702.

Arriagada, I. (1997). Políticas sociales, familia y trabajo en la América Latina de fin de siglo. Santiago de Chile: Naciones Unidas, 
Comisión Económica para América Latina y el Caribe.

Avedaño, R., \& Minujin, A. (1988). Una escuela diferente. La Habana Cuba: Pueblo y Educación.

Ayala, H., Pedroza, F., Morales, S., Chaparro, A., \& Barragán, N. (2002). Factores de riesgo, factores protectores y generalización del comportamiento en una muestra de niños en edad escolar. Salud Mental, 25, 27-40.

Barba-Solano, C. (2012). Reseña de «Encuesta Nacional sobre Discriminación en México/ ENADIS 2010. Resultados sobre zonas metropolitanas de México, Guadalajara y Monterrey» del Consejo Nacional para Prevenir la Discriminación. Espiral, 19, 261270.

Concha-Eastman, A., \& Krug, E. (2002). Informe mundial sobre la salud y la violencia de la OMS: Una herramienta de trabajo. Rev Panam Salud Publica/Pan Am J Public Health 12(4), 227-229.

Contini de González, E. N. (2008). Las habilidades sociales en la adolescencia temprana: Perspectivas desde la psicología positiva. Psicodebate, Cultura y Sociedad, 9, 45-63.

Elizalde, A. (2010). Estudio descriptivo sobre las estrategias de afrontamiento del bullying entre profesorado mexicano. Education \& Psychology, 8(20), 353-372.

Farrington, D. (1993). Understanding and preventing bullying. Crime and Justice, 17, 381458.

Fierro-Evans, M. C. (2013). Convivencia inclusiva y democrática: Una perspectiva para gestionar la seguridad escolar. Sinéctica, 40, 01-18.

Fried, S., \& Fried, P. (1996). Bullies \& victims: Helping your child trough the schoolyard battlefield. New York: Evans and Company Gómez-Palacios, J. (1991). Educar para la paz. Madrid: CCS.

Goodman, R., Ford, T., \& Richards, H. (2000). The development and well-being assessment: Description and initial validation of an integrated assessment of child and adolescent psychopathology. Journal of Child Psychology and Psychiatry, 41(5), 645-55.

Hinojosa, G. (2008). El tratamiento estadístico de las redes semánticas naturales. Revista Internacional de Ciencias Sociales y Humanidades, SOCIOTAM, 18(1), 133-154.

Hunter, C., \& Boyle, M. (2004). Appraisal and coping strategy use in victims of school bullying. British Journal of Educational Psychology, 74, 83-107.

Marín-Martínez,A., \& Reidl-Martínez, L. (2013). Validación Psicométrica del Cuestionario "Así nos llevamos en la Escuela" para evaluar el Hostigamiento escolar (bullying) en primarias. Revista Mexicana de Investigación Educativa, 18(56), 11-36.

Mendoza, B. (2014.) Bullying. Asambleas escolares para mejorar la convivencia escolar. D. F., México: Manual Moderno.

Menesini, E., Eslea, M., Smith, P. K., Genta, M. L., Giannetti, E., Fonzi, A., \& Costabile, A. (1997). Cross-national comparison of children's attitudes towards bully/victim problems in school. Aggressive Behavior, 23(4), 245-257. 
Olweus, D. (1973). Personality and aggression. En J. Coie \& D. Jensen (Eds.), Nebraska symposium on motivation (pp. 261-321). Lincoln: University of Nebraska Press

Olweus, D. (1978). Aggression in the schools: Bullies and whipping boys. Washington, DC: Wiley.

Olweus, D. (1993). Bullying at School. Malden, MA: Blackwell Publishing.

Olweus, D. (2001). Bullying at school: Tackling the problem. Organization for Economic Cooperation and Development, 25, 24-26

Organización Mundial de la Salud. (OMS, 2002). Informe mundial sobre la violencia y la salud: Resumen. Washington, D.C.: Organización Panamericana de la Salud.

Pedroza, J., Mendoza, G., \& Martínez, K. (2013). ;Auxilio! ;Mi hijo no trae manual!: Prácticas de crianza positiva, prevención de adicciones y bullying. D.F., México: Pax.

Pliego, C. F. (2013). Tipos de familia y bienestar de niños y adultos. México: Instituto de Investigaciones Sociales, UNAM.

Porcelli, G., \& Urpis, O. (2012). Children's Voices Qualitative National Report. Action co-funded by the European Union's Fundamental Rights and Citizenship Programme. Italy: Trieste.

Repetti, R. L., Taylor, S. E., \& Seeman, T. E. (2002). Risky families: Family social environments and the mental and physical health of offspring. Psychological bulletin, 128(2), 330-366.

Westhues, K. (junio, 2008). Críticas al movimiento anti-bullying. Ponencia presentada en la $6^{\mathrm{a}}$
Conferencia Internacional sobre Bullying, Montreal. Recuperada de http://www.kwesthues.com/e-bullyconf0806prelim.pdf

Zermeño, A., Arellano, A., \& Ramírez, A. (2005). Redes semánticas naturales: Técnica para representar los significados que los jóvenes tienen sobre la televisión, internet y expectativas de vida. Estudios sobre las Culturas Contemporáneas, 11(22), 305-334. 